\title{
Capsule Commentary on Danan et al., Does Motivation Matter? Analysis of a Randomized Trial of Proactive Outreach to VA Smokers
}

\author{
Amir Mohammad, MD, MPH, FACPM, FACOEM ${ }^{1,2}$ \\ 'Yale University School of Medicine, New Haven, CT, USA; ${ }^{2}$ VA Connecticut Healthcare System, New Haven, CT, USA.
}

J Gen Intern Med 31(8):927

DOI: $10.1007 / \mathrm{s} 11606-016-3715-1$

() Society of General Internal Medicine 2016

I $\mathrm{n}$ this multicenter randomized controlled trial, the authors examined the effectiveness of proactive smoking cessation intervention and its impact on veteran smokers' level of motivation to quit. ${ }^{1}$ Using the transtheoretical model of behavioral stages of change (SOC), they assessed veterans' readiness to quit smoking after categorizing them under precontemplation, contemplation, and preparation phases while receiving either usual or proactive care. SOC was assessed at baseline and follow-up with the 10-point Readiness to Quit Ladder (RQL).

Proactive care offered telephone or in-person smoking cessation counseling and access to cessation pharmacotherapy. The usual care group had access to smoking cessation services through the VA, but did not receive proactive outreach. The outcomes were self-reported 6- month abstinence and uptake of smoking cessation therapies and quit attempts at baseline and follow-up.

The study recruited 3006 veterans of diverse age, ethnicity, and socioeconomic status (SES) representing four different VA medical centers. The authors report a statistically significant result among smokers in contemplation and preparation phases achieving prolonged abstinence after proactive care. Smokers at all SOC receiving proactive care had statistically significant uptake of smoking cessation therapies. However, there was no difference in quit attempts between proactive and usual care groups.

The literature indicates that smoking is highly prevalent among veterans and remains a major cause of excess morbidity, mortality, and loss of productivity. ${ }^{2,3}$ Routine advice from providers increases the likelihood that a smoker will make a quit attempt. Studies indicate that the interventions that proactively offer evidence-based smoking cessation therapies to all smokers, regardless of SOC, may provide further opportunity for reducing smoking prevalence. ${ }^{4}$

Veterans with psychiatric disorders and other comorbidities smoke at higher rates, ${ }^{5}$ however, this information is not provided. With the exception of participation and recall biases, current findings are highly encouraging for clinicians.

Clinical decision support systems (CDSS) promote the use of best practices, condition-specific guidelines, and population-based management. By leveraging CDSS within the electronic medical record (EMR), VA providers can identify at-risk groups, and can provide evidence-based risk assessment and targeted intervention to our veteran smokers.

Future studies should utilize electronic tools, including CDSS, to enhance proactive outreach strategies while providing tobacco cessation therapy.

Corresponding Author: Amir Mohammad, MD, MPH, FACPM, FACOEM; Yale University School of Medicine, New Haven, CT, USA (e-mail: amir.mohammad@yale.edu).

\section{Compliance with Ethical Standards:}

Conflict of Interest: The author has no conflict with any of the material in this manuscript.

\section{REFERENCES}

1. Danan ER, Joseph AM, Sherman SE, et al. Does motivation matter? Analysis of a randomized trial of proactive outreach to VA smokers. J Gen Intern Med. 2016; doi: 10.1007/s11606-016-3687-1.

2. Brown DW. Smoking prevalence among US veterans. J Gen Intern Med. 2010;25(2):147-149.

3. Helyer AJ, Berehm WT, Perion L. Economic consequences of tobacco use for the Department of Defense, 1995. Mil Med. 1998;163(4):217-221.

4. Aveyard P, Begh R, Parsons A, et al. Brief opportunistic smoking cessation interventions: a systematic review and meta-analysis to compare advice to quit and offer to assistance. Addiction. 2012;107:1066-73.

5. MCFall M, Saxon AJ, Thompson CE, Yoshimoto D, Malte C, StraitsTröster K, Kanter E, Zhou XH, Dougherty CM, Steele B. Improving the rates of quitting smoking for veterans with posttraumatic stress disorder. Am J Psychiatry. 2005; 162(7):1311-1319.

Published online April 29, 2016 